\title{
The Best Bone of Acetabulum for Cup Component Placement in Total Hip Arthroplasty in Patients with Crowe Type I-III Dysplastic Hips: A Computer Simulation Study
}

\author{
Linli Zheng ${ }^{1 *}$, Yangyang Lin $^{2 \dagger}$, Xiaoyan Zhang ${ }^{3 \ddagger}$, Qianhui Ling ${ }^{4 \S}$, a \\ Weiming Liao ${ }^{1 * * *}$ and Peihui $\mathrm{Wu}^{1, \dagger \dagger}$ \\ ${ }^{1}$ Joint Surgery, First Affiliated Hospital of Sun Yat-sen University, Guangzhou, China \\ ${ }^{2}$ Rehabilitation Medicine, the Six Affiliated Hospital, Sun Yat-sen University, Guangzhou, China \\ ${ }^{3}$ College of Computer Science \& Software Engineering, Shenzhen University, Shenzhen, China. \\ ${ }^{4}$ Zhongshan Medical School, Sun Yat-sen University, Guangzhou, China. \\ alexandracefoxmail.com, linyy49@mail.sysu.edu.cn, \\ xyzhang15@szu. edu.cn, lingqh@outlook.com, 13809775039@163.com, \\ wupeihui@mail2.sysu.edu.cn
}

\begin{abstract}
For cup implantation in dysplastic acetabulum, the vertical height of the cup center (V-HCC) should be carefully and precisely controlled in order to achieve sufficient host bone-cup coverage (BCC), but excessively superior placement of the cup should be avoided. Using computer software, pelvis models were separately reconstructed in 51 patients (61 hips) with severe osteoarthritis secondary to Crowe type I-III hips. Acetabular height and doom thickness were measured on the mid-acetabular coronal cross section. V-HCC was defined as the vertical distance from the cup center to the interteardrop line (ITL). In the cup implantation simulation, the cup was placed at the initial preset position, with a V-HCC of $15 \mathrm{~mm}$, and moved proximally by $3-\mathrm{mm}$ increments. At each level, the BCC was automatically calculated by computer. There were no significant between-group differences in maximum thickness of the acetabular doom; however peak bone stock values were obtained at heights of $41.63 \mathrm{~mm} \pm 5.14 \mathrm{~mm}$ (Crowe type I), $47.58 \mathrm{~mm} \pm 4.10 \mathrm{~mm}$ (Crowe type II), and $55.78 \mathrm{~mm} \pm 3.64 \mathrm{~mm}$ (Crowe
\end{abstract}

\footnotetext{
* Designed and perform the study, do the statistical analysis and wrote the manuscript

$\dagger$ Designed the study and revised the draft

\$Provided the software for morphological analysis

${ }^{\S}$ Collect clinical data and do statistical analysis

${ }^{* *}$ Reviewed and revised the draft

${ }^{\dagger}$ Designed the study, provided the software, wrote, revised and edited the manuscript
} 
type III) above the ITL. At $15 \mathrm{~mm}$ of HCC, BCC was $79 \% \pm 7 \%$ (Crowe type I), 74\% $\pm 9 \%$ (Crowe type II), and $61 \% \pm 6 \%$ (Crowe type III). In order to achieve $80 \%$ of the BCC, the evaluation distance was $1.33 \pm 1.62 \mathrm{~mm}(95 \% \mathrm{CI} 0.67-2.01), 3.32 \pm 2.94 \mathrm{~mm}(95 \% \mathrm{CI}$ 1.94-4.69), and 9.68 $\pm 3.91 \mathrm{~mm}$ (95\% CI 7.60-11.77) for Crowe type I, II, and III hips, respectively. Acetabular bone stock for cup placement correlates with the degree of hip dysplasia in patients. During acetabular reconstruction in Crowe type I, II, or III hips, slightly superior placements, $<25 \mathrm{~mm}$ from the ITL, retained sufficient bone coverage.

\section{Introduction}

Total hip arthroplasty (THA) is the standard, efficacious treatment for advanced degenerative arthritis in patients with developmental dysplasia of the hip (DDH) (Rogers, Garbedian, Kuchinad, Backstein, Safir, \& Gross, 2012). Dysplastic hips can be classified according to the severity of observed anatomic abnormalities. Cameron et al. found that the Crowe classification system can predict surgical complexity and the likelihood of complications (Cameron, Botsford, \& Park, 1996). In severe cases of $\mathrm{DDH}$, acetabular reconstruction is technically demanding because the acetabular concavity is shallow (Hartoflakidis, Yiannakopoulos, \& Babis, 2008).

Upward placement of the cementless cup component may allow for better bone stock than "an anatomic position," especially in Crowe type II or III hips (Xu, Qu, Li, Mao, Yu, \& Zhu, 2017). The combined positive and negative results of the superiorly placed cementless components are intriguing. For cup implantation in a dysplastic acetabulum, the vertical height of the cup proximal placement should be carefully and precisely controlled in order to achieve sufficient host bone-cup coverage, but excessively superior placement of the cup should be avoided. In this context, we present a novel threedimensional (3D) morphological measurement method for evaluating the bone stock of the dysplastic acetabulum in Crowe type I-III hips and to investigate the relationship between the hip center height and host bone-cup coverage in patients with hip dysplasia using computer simulation software.

\section{Method}

\subsection{Patients}

A total of 51 patients (61 hips) were included,10 of whom had bilateral involvement and 41 with unilateral hip involvement. According to the Crowe classification system, 25 were Crowe type I, 20 were Crowe type II, and 16 were Crowe type III.

\subsection{Acetabular Morphology Evaluation and Measurement}

Hip CT scan was performed on each patient and a 3D pelvis model was separately reconstructed using BOHOLO software (version, Fengsuan Ltd. Shanghai China). 3D pelvis models were imported into a home-developed software in stereolithographic (STL) format. The pelvis model was adjusted into neutral position and assessed the bone stock distribution on the acetabular mid-coronal cross section. (Figure 1 a-f). 


\subsection{Simulating Implantation of the Prosthetic Acetabular Component}

The vertical height of the cup center (V-HCC) was defined as the vertical distance from the center of the cup component to the ITL. The simulated acetabular implantation was performed using BOHOLO software by placing the cup component at the initial preset position, with a V-HCC of 15 $\mathrm{mm}$ (Lucaciu, Berce, Lucaciu, \& Cosma, 2018), a cup inclination of $40^{\circ}$, and an anteversion of $15^{\circ}$. Thereafter, the cup was stepwisely moved proximally by $3 \mathrm{~mm}$ increments. The host bone-cup coverage ratio was automatically calculated at each level by computer. (Figure 1. g-i)

\subsection{Statistical Analysis}

We compared differences among the different Crowe groups using the one-way ANOVA (analysis of variance) test, followed by the least significant difference (LSD) method for pairwise comparisons.

\section{Results}

\subsection{Morphological Analysis}

The maximum thickness of the acetabular roof bone stock was $44.15 \pm 6.75 \mathrm{~mm}$ in Crowe type I hips, $43.99 \pm 6.29 \mathrm{~mm}$ in Crowe type II hips, and $38.81 \pm 7.73 \mathrm{~mm}$ in Crowe type III hips. There were no significant between-group differences among Crowe type I-III hips. The height of the acetabulum in Crowe type III hips $(55.78 \pm 3.64 \mathrm{~mm})$ was significantly larger than Crowe type I $(41.63 \pm 5.14 \mathrm{~mm}$, $\mathrm{p}<0.01)$ and Crowe type II hips $(47.58 \pm 4.10 \mathrm{~mm}, \mathrm{p}<0.01)$.

\subsection{Host Bone-Cup Coverage Ratio Versus the Height of the Cup Center From the ITL}

At the initial cup center position, which was $15 \mathrm{~mm}$ above ITL, the host bone-cup coverage ratios were $79 \% \pm 7 \%$ (Crowe type I), 74\% $\pm 9 \%$ (Crowe type II), and $61 \% \pm 6 \%$ (Crowe type III). In order to achieve $80 \%$ of the coverage ratio, the evaluation distance was $1.33 \pm 1.62 \mathrm{~mm}$ (95\% CI 0.67-2.01), $3.32 \pm 2.94 \mathrm{~mm}(95 \%$ CI 1.94-4.69), and $9.68 \pm 3.91 \mathrm{~mm}$ (95\% CI 7.60-11.77) or Crowe type I, II, and III hips, respectively. The coverage ratios increased to peak values of $96.7 \pm 2.2 \%$ at 21 to $24 \mathrm{~mm}$ above the ITL (Crowe type I), 96.7 $\pm 2.5 \%$ at 24 to $27 \mathrm{~mm}$ above the ITL (Crowe type II), and $88.1 \pm 7.2 \%$ at 30 to $33 \mathrm{~mm}$ above the ITL (Crowe type III). (Figure 2) 


\section{Discussion}

This 3D computer simulation study of cup component placement in patients with Crowe type I-III dysplastic hips demonstrated that slightly superior placements of $<25 \mathrm{~mm}$ from the ITL retained sufficient bone coverage.

Sufficient host bone coverage is crucial for reliable cup fixation. Due to the highly sloped acetabular roof of the dysplastic hip, the surgeon may believe that inferior placement of the hip center may produce insufficient host bone coverage and compromised initial cup stability. High cup component placement was necessary for sufficient bone coverage. However, this surgical technique also introduces the possibility of over-elevation (Watts, Abdel, Hanssen, \& Pagnano, 2016 ) (Nie, Pei, \& Li, 2014). Our results showed that a $\mathrm{V}-\mathrm{HCC}$ of $15 \mathrm{~mm}$ retained host bone-cup coverage by $79 \% \pm 7 \%, 74 \% \pm 9 \%$, and $61 \% \pm 6 \%$ in Crowe type I, II and III hips respectively. Even if the bone coverage was set to $80 \%$, the V-HCC was $16.33 \pm 1.62 \mathrm{~mm}, 18.32 \pm 2.94 \mathrm{~mm}$, and $24.68 \pm 3.91 \mathrm{~mm}$ for Crowe type I, II and III hips. Past studies indicated that a V-HCC $<25 \mathrm{~mm}$ was associated with long-term implant survival, lower joint dislocation risk, early recovery of abductor muscle moment, and establishment of an impingement-free range of joint motion (Komiyama, Fukushi, Motomura, Hamai, Fuji, \& Nakashima, 2018) (Komiyama, Nakashima, Hirata, Hara, Kohno, \& Iwamoto, 2016) (Georgiades, Babis, Kourlaba, \& Hartofilakidis, 2010) (Fukushi, Kawano, Motomura, Hamai, Kawaguchi, \& Nakashima, 2018).

Nawabi et al. reported no cases of aseptic loosening at a mean follow-up of 12 years. The mean VHCC was $17.3 \mathrm{~mm}, 25.6 \mathrm{~mm}$, and $30.3 \mathrm{~mm}$ in Crowe type I, II and III hips (Nawabi, Meftah, Nam, Ranawat, \& Ranawat, 2014). Additionally, Kaneuji et al. reported the mean V-HCC was $21.8 \pm 1.3 \mathrm{~mm}$, $28.2 \pm 3.4 \mathrm{~mm}$, and $32.3 \pm 5.6 \mathrm{~mm}$ in Crowe type I,II and III hips respectively. There were no acetabular failures at a minimum of 10 years (Kaneuji, Sugimori, Ichiseki, Yamada, Fukui, \& Matsumoto, 2009). In these two clinical studies, among different Crowe groups, hip centers were placed about 5-10 mm higher than those in the current study. It is possible that the acetabular reaming may not be fully medialized or the surgeons may underestimate the actual bone coverage during the operation. Surgeons will naturally tend to upwardly file the acetabulum to obtain more sufficient bone coverage. A 3D computer stimulation based on CT-scan images could provide accurate information pertaining to optimal cup component position and corresponding bone coverage. This could help surgeons evaluate acetabular bone stock distribution; make intraoperative decisions regarding cup size, hip center height.

In summary, in patients undergoing THA secondary to DDH, the bone stock distribution of the acetabulum, which varies according to dysplasia severity, should be taken into account when reaming the socket during acetabular reconstruction, slightly superior placements, $<25 \mathrm{~mm}$ from the ITL, retained sufficient bone coverage. 

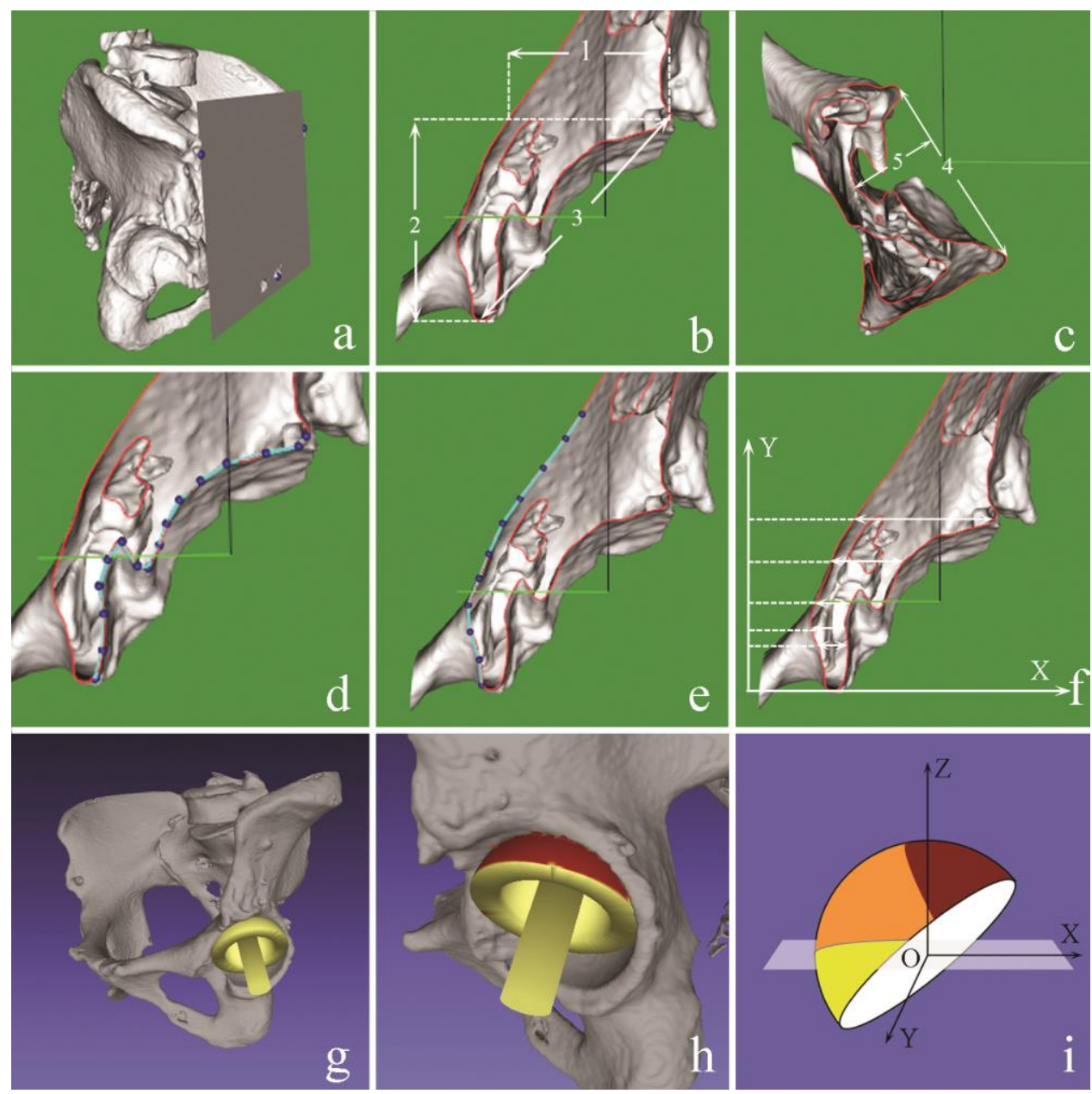

Figure 1 Acetabular morphology measurement and simulating implantation of the cup component a. Align the pelvis on the anterior pelvic plane (APP); b. Measurements on the mid-coronal section: 1. Acetabular roof bone stock; 2. Acetabular height; 3. Superioinferior diameters; c. Measurements on the mid-horizontal section. 4. Anterioposterior diameter; 5. Acetabular depth. d,e. Polyline outlines of the cortical bone of the acetabulum were drawn on the mid-coronal section; f. Calculation of the medial wall thickness at $0.5 \mathrm{~mm}$ intervals above the anatomic ITL; g. Simulation of the cup placement (Inclination $=40^{\circ}$; Anteversion $\left.=15^{\circ}\right)$; $\mathbf{h}$. The uncovered area on the cup (red portion); i. Calculation of the cup coverage. surface area of the covered portion over the surface of superior portion of the virtual cup(orange color)/total surface area of the superior portion of virtual cup(red portion+orange portion) $\times 100 \%$. 


\section{Distance from Teardrop / $\mathrm{mm}$}

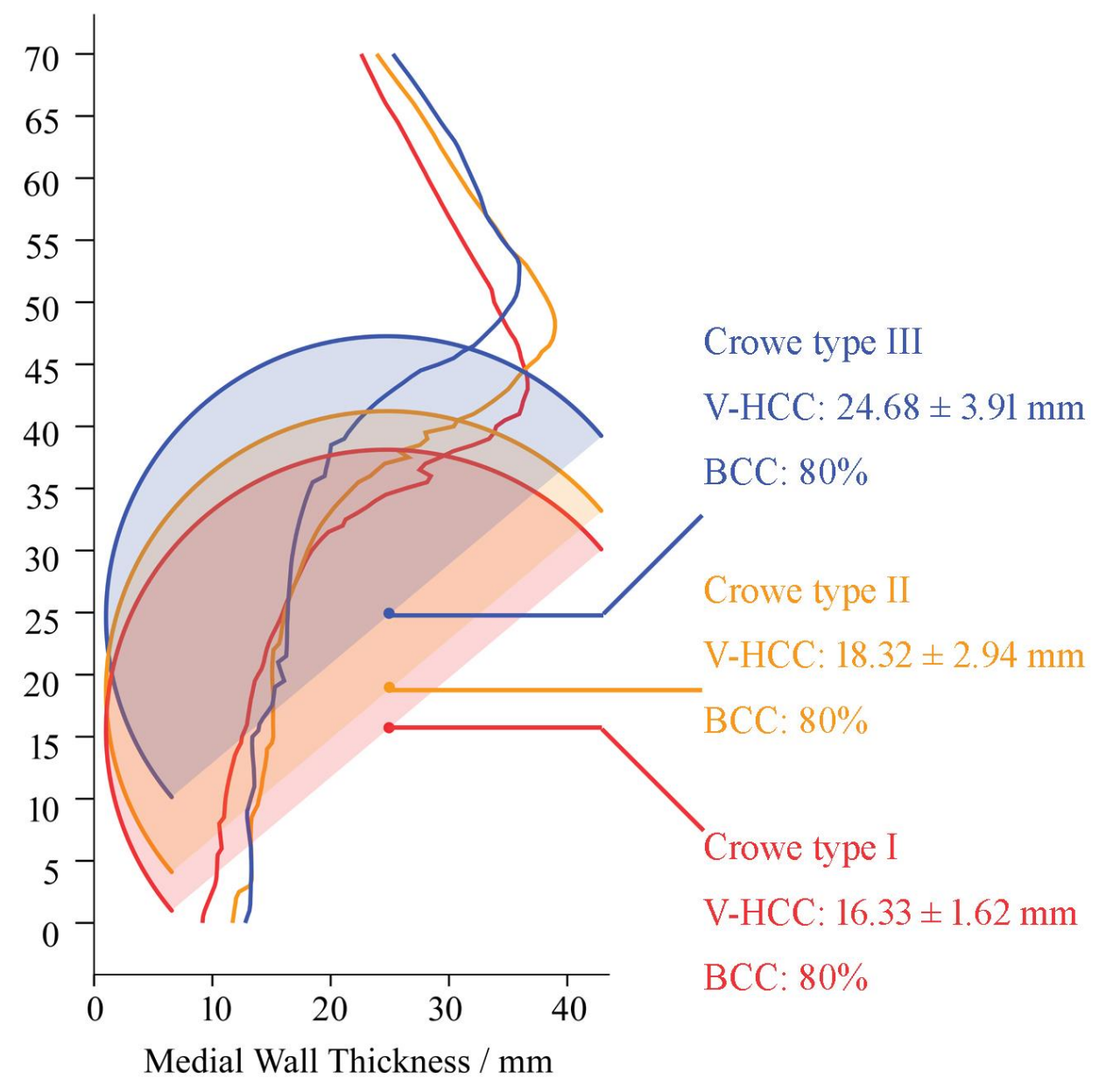

Figure 2 To achieve $80 \%$ of the BCC, the V-HCC was $16.33 \pm 1.62 \mathrm{~mm}, 18.32 \pm 2.94 \mathrm{~mm}$, and $24.68 \pm 3.91 \mathrm{~mm}$ for Crowe type I, II, and III hips, respectively. BCC: Host bone-cup coverage; V-HCC: vertical distance from cup center to the ITL.

\section{References}

Cameron, H., Botsford, D., \& Park, Y. (1996, Aug). Influence of the Crowe rating on the outcome of total hip arthroplasty . J Arthroplasty., pp. 11(5):582-7.

Fukushi, J., Kawano, I., Motomura, G., Hamai, S., Kawaguchi, K., \& Nakashima, Y. (2018, Dec). Does hip center location affect the recovery of abductor moment after total hip arthroplasty? . Orthop Traumatol Surg Res, pp. 104(8):1149-1153. 
Georgiades, G., Babis, G., Kourlaba, G., \& Hartofilakidis, G. (2010, Oct). Effect of cementless acetabular component orientation, position, and containment in total hip arthroplasty for congenital hip disease. J Arthroplasty, pp. 25(7):1143-50.

Hartoflakidis, G., Yiannakopoulos, C., \& Babis, G. (2008, Apr). The morphologic variations of low and high hip . Clin Orthop Relat Res, pp. 466(4):820-4.

Kaneuji, A., Sugimori, T., Ichiseki, T., Yamada, K., Fukui, K., \& Matsumoto, T. (2009, Feb). Minimum ten-year results of a porous acetabular component for Crowe I to III hip dysplasia using an elevated hip center. . J Arthroplasty, pp. 24(2):187-94.

Komiyama, K., Fukushi, J., Motomura, G., Hamai, S., Fuji, M., \& Nakashima, Y. (2018, Sep). Does high hip centre affect dislocation after total hip arthroplasty for developmental dysplasia of the hip? Int Orthop, p. 24.

Komiyama, K., Nakashima, Y., Hirata, M., Hara, D., Kohno, Y., \& Iwamoto, Y. (2016, Oct). Does High Hip Center Decrease Range of Motion in Total Hip Arthroplasty? A Computer Simulation Study. J Arthroplasty, pp. 31(10):2342-7.

Lucaciu, O., Berce, C., Lucaciu, D., \& Cosma, D. (2018, Jun). Current methods of preventing aseptic loosening and improving osseointegration of titanium implants in cementless total hip arthroplasty: a review. J Int Med Res., pp. 46(6):2104-2119..

Nawabi, D., Meftah, M., Nam, D., Ranawat, A., \& Ranawat, C. (2014, Feb). Durable fixation achieved with medialized, high hip center cementless THAs for Crowe II and III dysplasia. Clin Orthop Relat Res, pp. 472(2):630-6.

Nie, Y., Pei, F., \& Li, Z. (2014, July). Effect of high hip center on stress for dysplastic hip. Orthopedics, pp. 37(7):e637-43.

Rogers, B., Garbedian, S., Kuchinad, R., Backstein, D., Safir, O., \& Gross, A. (2012, Oct 3). Total hip arthroplasty for adult hip dysplasia. J Bone Joint Surg Am., pp. 94(19):1809-21.

Watts, C., Abdel, M., Hanssen, A., \& Pagnano, M. (2016, Jun 1). Anatomic Hip Center Decreases Aseptic Loosening Rates After Total Hip Arthroplasty with Cement in Patients with Crowe Type-II Dysplasia: A Concise Follow-up Report at a Mean of Thirty-six Years. J Bone Joint Surg Am., pp. 98(11):910-5.

Xu, J., Qu, X., Li, H., Mao, Y., Yu, D., \& Zhu, Z. (2017, Apr). Three-Dimensional Host Bone Coverage in Total Hip Arthroplasty for Crowe Types II and III Developmental Dysplasia of the Hip. $J$ Arthroplasty, pp. 32(4):1374-1380. 